\title{
Complexity and dynamic characteristics of a new discrete-time hyperchaotic model
}

\begin{abstract}
Based on two of the existing one-dimensional chaotic maps and the two-dimensional Hénon map, a new two-dimensional Hénon-Gaussian-Sine model (2D-HGSM) is proposed. Basic dynamic characteristics of the 2D-HGSM are studied from the following three aspects: trajectory, bifurcation diagram and Lyapunov exponents. The complexity of 2D-HGSM is investigated by means of Approximate entropy. Performance evaluations show that the 2DHGSM has higher complexity level, better ergodicity, wider chaotic and hyperchaotic region than different chaotic maps. Furthermore, the 2D-HGSM exhibits a qualitatively different chaotic behavior with respect to the variation of its corresponding parameters. Therefore, the 2D-HGSM has good application prospects in secure communication.
\end{abstract}

Keyword: Hyperchaotic; Lyapunov exponents; Approximate entropy; Complexity analysis 